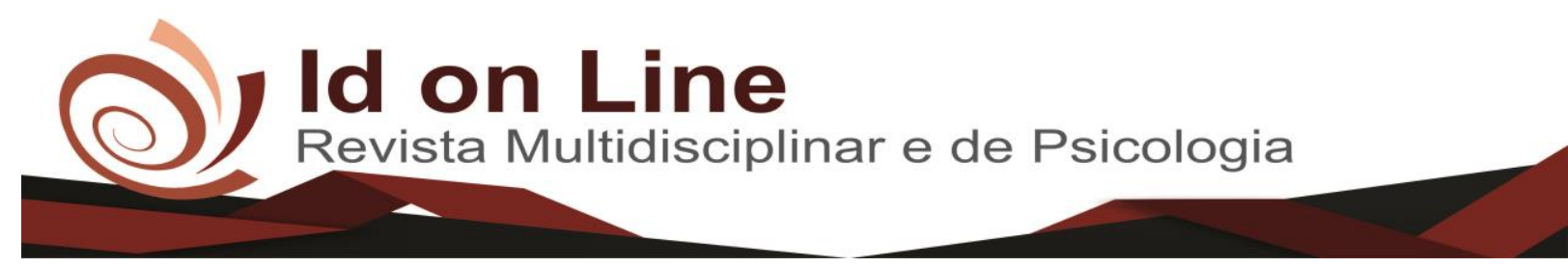

Comment

\title{
Webquest e mobile-learning como métodos pedagógicos de ensino e aprendizagem: um novo olhar?
}

\author{
Gilmária Henllen Gondim Gomes ${ }^{2}$; Liromaria Maria de Amorim² ; Micaelle Nayara Dias Rodrigues ${ }^{4}$; \\ Jailma dos Santos Barbosa ${ }^{4}$; Gislene Farias de Oliveira ${ }^{5}$
}

\begin{abstract}
Resumo: O presente estudo discute a inclusão das TIC no processo educacional. Estes implicam em reflexões importantes sobre o impacto das novas tecnologias no âmbito da educação. As ferramentas tecnológicas enquanto estratégias educacionais exigem um mais aguçado planejamento das aulas, bem como dá ênfase ao papel do professor ante a aprendizagem dos alunos. A inserção da webquest e da mobile lerning nas aulas trouxe ganho para ambos os lados, desde uma melhoria na motivação, até um maior envolvimento de todos os atores no processo de ensino, na construção e aquisição dos conteúdos.
\end{abstract}

Palavras-Chave: Webquest, mobile-learning, Metodologias pedagógicas.

\section{Webquest and mobile-learning as Pedagogical methods of Teaching and Learning: A new look?}

\begin{abstract}
The present study discusses the inclusion of ICT in the educational process. These imply important reflections on the impact of new technologies on education. Technological tools as an educational strategy require a sharper class planning, as well as emphasizing the teacher's role in student learning. The insertion of webquest and mobile lerning in classes brought gains to both sides, from an improvement in motivation to greater involvement of all actors in the teaching process, in the construction and acquisition of content.
\end{abstract}

Keywords: Webquest, mobile-learning, Pedagogical methodologies.

\footnotetext{
${ }^{1}$ Graduação em Direito pela Faculdade Paraíso de Juazeiro do Norte e graduação em Licenciatura Plena Em Pedagogia pela Universidade Federal da Paraíba. Especialização em Psicopedagogia pela Faculdades Integradas de Patos. Atualmente é PróReitora adjunta da PROGEP/UFCA da Universidade Federal do Cariri e Servidor técnico-administrativo da Universidade Federal do Cariri. Contato: gilmaria.gondim@ufca.edu.br;

${ }^{2}$ Graduação em Saúde Pública e Pedagogia, pela Universidade Regional do Cariri - URCA; Especialização em Educação Infantil e Ensino Fundamental pela Faculdade de Juazeiro do Norte - FJN; Especialização em Educação Infantil pela Universidade Regional do cariri - URCA. Contato: lirinhamor@gmail.com.

${ }^{3}$ Graduação em Administração pelo centro Universitário Doutor Leão Sampaio - UNILEÃO. Especialização em Recursos Humanos pela Faculdade de Juazeiro do Norte - FJN. Contato: micaellenayara@ hotmail.com;

${ }^{4}$ Mestre em Ensino na Saúde pela Universidade Estadual do Ceará - UECE. Graduada em Enfermagem. e Pedagogia. Especialista em Gestão em Saúde, Especialista em Auditoria em Saúde, Especialista em Enfermagem do Trabalho, Especialista em Saúde da Família, Especialista em Gestão Escolar. E-mail: jailmaestre@ hotmail.com;

${ }^{5}$ Doutorado em psicologia Social pela Universidade Federal da Paraíba. Docente na Faculdade de Madicina da Universidade Federal do Cariri. Contato: gislenefarias@gmail.com.
} 


\section{Introdução}

No passado, os principais métodos inovadores de aprendizagem envolviam estudos em ambientes fora de sala de aula, como museus, jardins, zoológicos etc, visto que a interação com esses ambientes variados colabora para uma aprendizagem mais completa, que além de melhorar o desemprenho de aprendizagem do aluno (LIU, TAN, \& CHU, 2009), e sua criatividade, estimulavam o desenvolvimento de estratégias sociais e cognitivas mais complexas, necessárias a sua interação com os outros alunos e com o mundo em geral.

Em 1969, Dale apresentou estudo que revelou que, quando os educandos participavam de experiências concretas, ou diretas, relacionadas com a vida real; planejadas, com o uso de métodos interativos; ou dramáticas, com base em dramatizações, elas aprendiam de forma mais eficaz do que quando apenas envolvidas com o estudo em abstrato. Nesse sentido, a educação ao ar livre, usando locais naturais ou construídos, demonstrou ser potencializadora do ensino, permitindo novas oportunidades de expansão do conhecimento. Isso, em razão das novas experiências contextuais obtidas com a interação com os novos ambientes.

Hodiernamente, pesquisas apontam que o uso da tecnologia pode ajudar o educando a aumentar seus conhecimentos nas mais diversas áreas, aumentando seu conhecimento, bem como motivando-o a participar das atividades de aprendizagem (AKKERMAN et al., 2009; CHEN, CHANG, LI e LI, 2008). O amplo e facilitado acesso à computação e demais tecnologias, possibilitam o contato com novas experiências de aprendizado, podendo configurar uma substituição viável para as antigas instruções ao ar livre, por também constituir ambiente de análise, interação e pesquisa. São ambientes em que o Webquest tem se mostrado eficiente, principalmente por se configurar numa ferramenta importante ao ensino à distância.

O Webquest trata-se de uma atividade desenvolvida com os alunos, direcionada a investigação de informações, por meio da qual terão que utilizar recursos da internet, que podem envolver desde uma simples pesquisa em um sítio eletrônico, até uma vídeo conferência (DODGE, 1997). A Webquest pode ser interpretada como sendo um minimundo, no qual os educandos irão explorar as tarefas em um ambiente de pesquisa interativo e, com maior densidade de informações. 
Consiste em ferramenta capaz de aprimorar a aprendizagem dos educandos, por meio da promoção de um ensino cooperativo em equipe e, ao mesmo tempo, com a atribuição de responsabilidades individuais para os alunos, em que os educandos debatem questões, discutem assuntos importantes, participam de simulações, de forma que estabelecem uma conexão e envolvimento mais forte com o que está sendo estudado.

Assim, o uso dessa ferramenta, não se limita a busca de novas informações na internet, mas também colabora para a promoção da habilidade analítica e crítica dos estudantes, com vistas a avaliar e solucionar problemas, pois é incentivado o debate de questões significativas e participação em simulações, o que os conecta e envolve (WATSON, 1999).

Pesquisadores tem analisado a possibilidade do uso do Webquest em celulares, para fins de verificar os impactos positivos e negativos que podem decorrer dessa estratégia. Nesse sentido, tem-se verificado que a utilização de smartphones no processo de aprendizado tem colaborado para um aumento no desempenho dos estudantes (CHEN, CHANG, LIN e YU, 2009; LIU, TAN, e CHU, 2009). O celular é capaz de servir de guia para o educando, ajudandoo a ter um melhor conhecimento ambiental e geográfico, bem como melhorando sua motivação para aprendizagem devido ao estímulo da curiosidade e, do interesse em participar desse novo método.

Com base nisso, o presente estudo teve por escopo analisar a utilização das ferramentas Webquest e mobile lerarning como mecanismo pedagógico de ensino e aprendizagem nas escolas, e como ferramenta tecnológica de auxílio para o trabalho de conteúdos educacionais.

\section{A ferramenta do Webquest}

É indubitável que cada vez mais as inovações tecnológicas fazem parte do cotidiano das pessoas, principalmente por ter ampliado e incrementado os recursos de comunicação e de pesquisa. Segundo Kenski (2003, p.21) a evolução da tecnologia não está restrita aos bens materiais, isto porque também pode modificar não só a forma de pensar e agir das pessoas, como também o modo de interagir e de adquirir novos conhecimentos, principalmente porque a tecnologia tem colaborado para aumentar o acesso a informação, o que simplifica a aprendizagem. 
Conforme ensina Ramos (2008, p. 5), as novas Tecnologias de Informação e Comunicação (TIC) aumentaram o trânsito das informações, especialmente em razão da digitalização dos conteúdos, que facilitou a transmissão desses. Sobre esse tema, Rosita Sarita de Araújo (2005, p. 23-24) comenta que:

\footnotetext{
O valor da tecnologia na educação é derivado inteiramente de sua aplicação. Saber direcionar o uso da Internet na sala de aula deve ser uma atividade de responsabilidade, pois exige que o professor preze, dentro da perspectiva progressista, a construção do conhecimento, de modo a contemplar o desenvolvimento de habilidades cognitivas que instigam o aluno a refletir e compreender, conforme acessam, armazenam, manipulam e analisam as informações que sondam na Internet.
}

Nesse diapasão, a internet tem se demonstrado um recurso de grande valia para a pedagogia, colaborando para a difusão do conhecimento e como meio facilitador do processo educativo. Para tanto, o professor, enquanto mediador do ensino, deve selecionar as tecnologias que mais se adequem a sua didática e metodologia de ensino, para fins de utilizar os recursos tecnológicos de uma forma favorável para a instituição de ensino (SILVA; FERRARI, 2009, p. 2).

Em 1995, o professor Bernie Dodge, apresentou uma proposta metodológica pautada no uso da internet, a qual denominou de Webquest, sendo esta uma atividade investigativa na qual os educandos adquirem conhecimentos com o uso da internet. O Webquest consiste em uma investigação orientada com o uso dos recursos da Internet e complementados por videoconferências.

O Webquest consiste em ferramenta que pode ser interpretada da seguinte forma: web, porque utiliza um sítio eletrônico e a internet; e quest, porque visa desvendar um questionamento prévio, por meio de uma exploração investigativa realizada na internet (Chang \& Chang, 2007).

Trata-se de método de pesquisa que pode ser curto, realizado em até três aulas, cujo objetivo é a aquisição de um conhecimento, onde a Webquest possibilitará o acesso a uma gama de informações para esse fim; ou longo, que vai de uma a quatro semanas de aulas, que visará o refinamento do aprendido, momento em que será necessário que o aluno realize uma análise aprofundada dos conhecimentos, de forma a criar uma conexão entre o aprendido e os demais conteúdos disponíveis na internet ou fora dela (DODGE, 1996, p. 1). 
O uso da ferramenta do Webquest no ambiente escolar demanda que o professor realize cuidadosamente o planejamento dos conteúdo a serem ensinados e das atividades envolvidas, com uma adequação espaço-tempo e que exijam uso da internet de forma interessante para a realização da pesquisa, isto porque uma atividade desinteressante, sem apropriação do conteúdo ou com vistas ao estudo de conceitos vagos e rasos, pode ser algo desmotivador para o educando.

Dodge aponta seis elementos que devem ser observados na estrutura e utilização da Webquest, sendo estes: (i) introdução geral das orientações para uso ferramenta; (ii) definição da tarefa a ser realizado, devendo essa ser algo interessante; (iii) fontes informacionais necessárias; (iv) passo-a-passo a ser seguido para a realização da tarefa; (v) direcionamento acerca da melhor forma de fichar as informações adquiridas; e (vi) demonstração do aprendizado ao aluno, de forma a encoraja-lo a levar a expandir a experiência ou repeti-la. Já o Ministério da Educação (2015) complementou e elencou sete seções ideais para o uso da ferramenta, quais sejam: (i) introdução, (ii) Tarefa, (iii) Processo, (iv) Recursos, (v) Orientações, (vi) Avaliação e (vi) Conclusão.

A ferramenta é capaz de criar condições favoráveis de aprendizagem por meio do uso de recursos interativos presentes ou não na academia, de forma colaborativa ou individual, de acordo com a disciplina a ser lecionada e com as necessidades elencadas pelo educador (BARRROS, 2005, p. 1-18), onde o docente poderá mixar mais um de um modelo educativo na elaboração do seu método de ensino e aprendizagem.

Nesse sentido, consiste em instrumento presente em uma plataforma online, que deve ser utilizado para fins de somar e colaborar para a promoção de uma aprendizagem significativa mais completa. Ou seja, não é um mecanismo que gera ensino-aprendizagem, no entanto, possibilita que o educando tenha acesso a uma gama mais ampla de informações (ARAÚJO, 2005, p. 32-33), o que acrescerá, indubitavelmente ao seu arcabouço de conhecimentos.

Para tanto, conforme ensina Kenski (2003, p. 46) é preciso que o educador se posicione como um parceiro dos alunos na busca do saber, e não como monopolizador do conhecimento, orientando os educandos acerca das diversas possibilidade de se alcançar os conhecimentos, incentivando uma postura investigativa e crítica.

Dessa forma, é necessário que o educador faça um planejamento adequado com base nas suas necessidades, para que seja possível utilizar a ferramenta e seus recursos para qualificar 
e impactar positivamente o processo de ensino, isto porque o uso do Webquest nas salas de aula deve ser realizado de forma a potencializar a aprendizagem dos educandos. Inclusive, deve-se atentar para o fato de que da mesma forma que a internet pode ser um meio interativo capaz de facilitar e motivar a aprendizagem, também pode configurar um ambiente dispersivo e inútil, que dificultará a coleta de dados e o estudo, situação que é desinteressante para a didática pedagógica. (PIMENTEL, 2007, p. 4).

\section{Utilização de smartphones como meio para uso do Webquest}

O constante avanço das tecnologias móveis tem possibilitado a criação de espaço para novas experiências de ensino q aprendizado que podem ocorrer em uma variedade de ambientes internos e externos (ROGERS ET AL, 2005). O aprendizado com o uso dos smartphones, tem sido intitulado de mobile-learning, tem sido vista como uma solução viável para a aplicação de novos métodos pedagógicos, especialmente em razão de sua fácil portabilidade, o que facilita o seu uso nos mais diversos locais, a interatividade intersocial, individualidade, assim como adequação a depender do contexto abordado.

Existem seis principais características de um processo de aprendizagem com uso do mobile-learning: (i) urgência de aprendizagem, (ii) vontade e iniciativa de adquirir um dado conhecimento; (iii) facilidade de mobilidade (iv) maior interatividade (v) particularidade da atividade a ser realizada; e (vi) integração do conhecimento buscado (CHEN ET AL, 2003). Ou seja, consiste em mecanismo projetado que um aluno individualmente, ou em pequenos grupos, possam solucionar uma questão-problema que lhe for posta, sendo a internet a principal ferramenta de busca por informações que será utilizada para tanto.

O benefício do uso do mobile-learning versa principalmente sobre a possibilidade de acúmulo de experiências e conteúdos de forma rápida, de forma que é possível alcançar um conhecimento integral e preciso em um menor espalho de tempo, bem como a viabilidade de todos os alunos conseguirem, concomitantemente, acessar, utilizar e experimentar os mesmos conteúdos disponíveis no ambiente virtual.

A vantagem mais significativa do Webquest é sua capacidade de promoção das habilidades de aprendizagem dos educandos, permitindo que estes se tornem mais capacitados 
para a busca de informações e resolução de problemas, quando desafiada sua capacidade intelectual e acadêmica. (Vidoni \& Maddux, 2002). Nesse sentido, o uso de telefones celulares é uma opção vantajosa, isto porque possibilitará a efetuação da pesquisa de forma mais célere e em qualquer local que o aluno estiver.

Conforme já comentado, Dogde (1995) descreve algumas etapas para o uso do Webquest, quais sejam: (i) traçar um objetivo, sendo este o momento em que o educador irá captar a atenção e o interesse dos educandos; (ii) definição das tarefas a serem implementadas, devendo conter entre elas uma uma folha de aprendizado, um briefing e a construção de um website; (iii) explicação dos processos envolvidos para o desembaraço das tarefas postas; (iv) estabelecimento dos recursos, ensinamento sobre os recursos existentes no site da Webquest, sendo este o principal local onde os alunos devem realizar suas pesquisas; (v) avaliação, momento em que serão analisados a qualidade dos trabalhos de pesquisa realizados pelos educandos com base em critérios pré-definidos; e (vi) conclusão, onde os professores irão resumir a atividade realizada para fins de reforçar o conhecimento perseguido, apontando, se possível, os pontos positivos e negativos de cada aluno, para que estes possam melhorar na próxima pesquisa.

Sendo assim, o uso do mobile-learning é algo que pode influenciar positivamente no processo de ensino e aprendizagem (KANUKA, ROURKE; LAFLAMME, 2007, p. 260-271). Ainda, os resultados relacionados ao desempenho de aprendizagem dos discentes tem alcançado níveis mais elevados com o uso da ferramenta. A qualidade das pesquisas realizadas pelos estudantes também tem aumentado (CHAN, 2007; GASKILL ET AL., 2006).

Muitos estudiosos acreditam que uma forma de adquirir um conhecimento é por meio da existência de um contexto real de interação social, no qual o educando será incitado a solucionar um problema com base nos conhecimentos que já possui e observado o contexto social em que está inserido (BILLETT, 1996; YOUNG, 1993), de onde poderá obter novos conhecimentos. Ou seja, o aprendizado é proveniente de um processo que envolve uma experiencia que irá aumentar, transformar ou aperfeiçoar conhecimentos que o indivíduo já possuía.

Uma análise realizada por CHANG ET AL (2011, p. 1228-1239), demonstrou que a associação do processo de aprendizado com o uso do mobile-learning e o Webquest tem sido uma combinação capaz de estimular a motivação dos alunos, melhorando sua participatividade 
e, consequentemente, sua aprendizagem, principalmente porque o grau de aproveitamento do discente é diretamente proporcional ao seu grau de participação nas atividades propostas. Inclusive, apontasse-se que a ferramenta do Webquest é importante forma de busca por informações relevantes em um pequeno espaço de tempo, configurando meio útil e eficaz, sendo aconselhado seu uso no estudo das mais diversas disciplinas.

Ademais, pesquisas apontam que o uso de multimídia no processo de ensino e aprendizagem aumenta a motivação e interesse dos educandos no que está sendo lecionado, o que interfere positivamente no seu grau de aprendizado (BARBOUR, RIEBER, THOMAS E RAUSCHER, 2009, p. 333-347). Na verdade, quanto maior o espectro de recursos que o estudante tiver disponibilizado para colaborar com seu estudo, melhor será o ânimo, a motivação, o desempenho e a aprendizagem do educando (GREENWALD et al, 1996).

Diante do exposto, a análise do uso do Webquest nos telefones celulares, consiste em tema que merece atenção especial, tendo em vista os poucos estudos que versam sobre o tema frente ao avanço tecnológico e cada vez maior dependência dos dispositivos móveis pelos seres humanos.

\section{Possibilidade de utilização da ferramenta Webquest como método de avaliação de ensino}

A proposta do uso do Webquest para fins de avaliação educacional, tem por objetivo de apresentar um novo modelo de avaliatório com base nas TIC, por meio do uso de sítios eletrônicos gratuitos existentes na internet para a promoção de práticas ativas de cunho didático. Nesse processo é importante utilizar os tópicos apresentados pelo Ministério da Educação para o uso da Webquest (BRASIL, 2015).

Busca-se adequar o ambiente virtual para que este seja espaço proporcionador incentivo ao espírito de pesquisa e de ensino (PIMENTEL, 2007, p. 4), por isso, a atividade deve ser elaborada de maneira a despertar no educando a atividade reflexiva para fins de desvendar como irá realizar e concluir a tarefa proposta.

Devem ser estabelecidos os critérios, como desempenho, participação e demonstração de aquisição de conhecimentos; bem como os principais objetivos a serem atingidos pelo aluno, para que esse alcance uma pontuação positiva ao final. 
Pesquisa recente realizada por alunos na Universidade Federal do Paraná acerca do tema, realizou uma análise das percepções dos alunos e professores sobre o uso da ferramenta como forma de avaliação pedagógica. O referido estudo revelou que quanto a atividade que foi realizada com o uso da ferramenta, $60 \%$ dos alunos sentiram-se satisfeitos, e 40\%, não gostaram do uso da Webquest para essa finalidade:

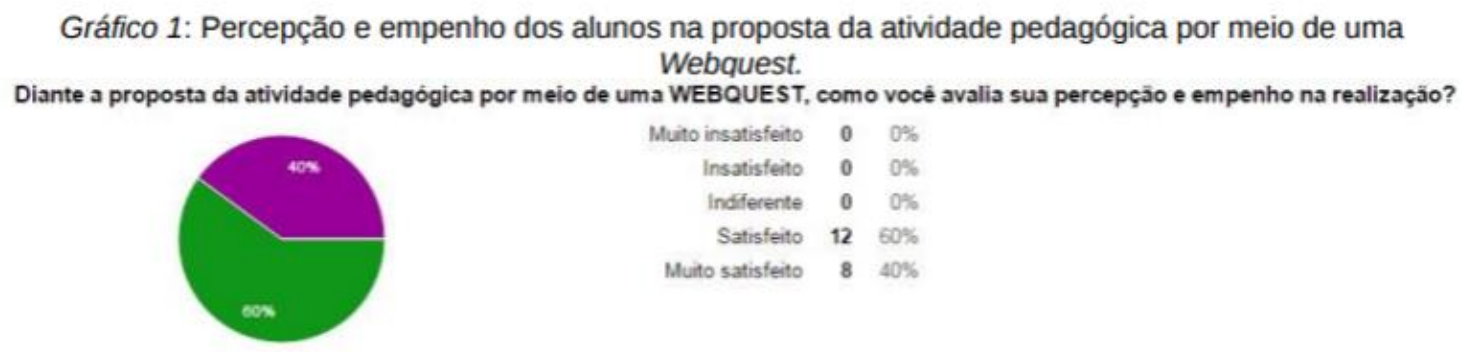

Fonte: http://www.periodicos.letras.ufmg.br/index.php/textolivre/article/view/11031

Com base no percentual de satisfação obtido, é possível verificar que o uso da Webquest despertou o interesse na realização da atividade pelos educandos, o que demonstra que as novas tecnologias são capazes de colaborar e suprir necessidades de ensino. Nesse sentido, quando os educadores oportunizam que os alunos tenham contato com a TIC no processo de ensinoaprendizagem, eles estão possibilitando a construção de conhecimentos de forma mais dinâmica e interessante, com o uso de novo com novas tecnologias, o que indica a elaboração de um novo modelo eficiente de ensino.

A mesma pesquisa avaliou a percepção dos alunos sobre os conteúdos trabalhados, por meio da qual foi possível verificar a qualidade do planejamento do professor para a realização da atividade na Webquest: 


\section{Gráfico 2: Percepção dos alunos quanto aos materiais disponibilizados na Webquest.}

\section{Quanto aos materiais disponibilizados on line para consulta e pesquisa:}
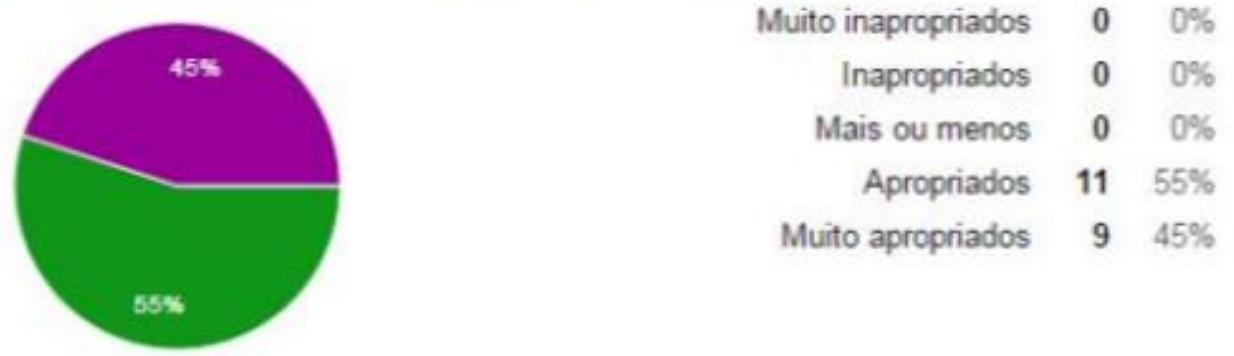

Fonte: http://www.periodicos.letras.ufmg.br/index.php/textolivre/article/view/11031

Conforme pode se inferir do gráfico, a maioria dos alunos consideraram a atividade apropriada. Com base na análise do envolvimento dos alunos com os conteúdo ministrados e do quantitativo de apropriação de conteúdo com o uso da ferramenta, pode-se verificar que a atividade foi adequada, tendo em vista que permitiu a compreensão e desembaraço da tarefa proposta de forma condizente.

Barros (2005) ensina que a tarefa deve ser elaborada de forma a preencher os objetivos propostos e previamente definidos pelo professor, juntamente com o intuito de propiciar e incentivar o envolvimento dos educandos na atividade trabalhada e estudada com a ferramenta, não devendo ser complicada de forma a configurar um óbice ao aprendizado. Essa parte da pesquisa é importante, isto porque a utilização desse inovador recurso tecnológico para fins pedagógicos, deve ter por escopo principal desenvolver a atividade crítica e reflexiva do educando.

Ademais, também colabora para o aprendizado do educador, de forma que esse consegue entender qual é a melhor forma de utilizar e gerir esse mundo de possibilidades, com fulcro de elaborar uma atividade investigativa e didática que potencialize o processo de ensino, tendo em vista a possibilidade da inclusão escolar da internet na avaliação dos alunos, e com meio de pesquisa e de consulta em classe.

A partir de uma abordagem problematizadora, o modelo webquest pode reduzir a probabilidade de desvios de rota nos hiperlabirintos da internet. Uma atividade bem orientada promove ganhos cognitivos significativos, pois envolve os alunos em um processo de busca, investigação, formulação de hipóteses e solução de problemas. Enfim, instiga-os a serem sujeitos ativos do processo de construção do próprio conhecimento (COELHO; VIDAL, 2009, p. 7). 
Nesse diapasão, é notório que os pontos positivos da ferramenta envolvem a possibilidade de o uso de várias fontes de aprendizagem como textos, imagens, vídeos, etc; configurando uma forma mais completa de aprendizado. O ponto negativo, conforme já comentado, é a possibilidade de dispersão do estudante devido aos demais conteúdos presentes na internet, o que pode desviar seu foco da atividade, dificultando a aquisição de conhecimentos.

Sendo assim, a Webquest trata-se de meio capaz de potencializar o estudo, em razão da interação com os mais diversos tipos de conteúdos, áreas de ensino e situações de aprendizagem. Para isso, é preciso que o educador planeje adequadamente uma estrutura coerente com as necessidades de ensino de seus alunos, buscando desenvolver o espírito investigativo e crítico dos estudantes, o que, ao final, colaborará para um aprendizado mais completo.

\section{Considerações Finais}

A ferramenta da Webquest, como recurso inovador que pode ser utilizado em métodos pedagógico ensino-aprendizagem, consiste em metodologia que utilizará a internet para a pesquisa ou o aprofundamento em determinado conteúdo ou campo de estudo. Por meio desta, o professor vai orientar os alunos acerca da melhor forma de se realizar uma pesquisa sistematizada e organizada de acordo com a tarefa a ser desenvolvida e os objetivos traçados, para que, ao final, apresentem uma síntese do estudado.

O uso da ferramenta desde os primeiros estágios escolares parece ser interessante por ser um método que busca desenvolver a capacidade cognitiva e crítica do aluno. Dessa forma, o desenvolvimento de atividades utilizando as TIC, consiste em método didático eficiente para tanto, contribuindo, consequentemente, para o aprimoramento do processo de aprendizagem.

Quando aplicado para fins avaliativos, a Webquest pode ser utilizada como uma atividade complementar das avaliações educacionais principais, visto que possibilita uma nova forma de aprendizagem, oportunizando momento interativos e novos desafios, fugindo do tradicionalismo. Logo, é método complementar pedagógico de grande valia, pois permite a 
expansão dos conhecimentos, com base em objetivos definidos e atividades a serem efetuadas; e no uso de outras fontes de pesquisa virtuais, como imagens e vídeos.

Com isso, é perceptível que o uso do Webquest, inclusive na forma de mobile-leraning, é capaz de entregar melhores resultados quando comparado ao método tradicional de ensino, atualmente defasado. Torna-se, então, de grande valia a inserção das TIC nos currículos escolares, no estudo e desenvolvimento dos mais diversos conteúdos e atividades de aprendizagem, visto que são capazes promover o desenvolvimento das capacidades dos intelectuais e pessoais dos alunos.

Como é perceptível a ferramenta trouxe ganho para ambos os lados, isto porque o processo de ensino passa a envolver tanto o educador, quanto o educando, na construção e aquisição do conteúdo. Logo, a Webquest, uma vez utilizada como método pedagógico, tem por base o uso de novos recursos tecnológicos para fins de ensino, o que demonstra a existência de uma nova ferramenta para a didática, não significando a substituição, tampouco exclusão de modelos e recursos já existentes, sendo, na verdade, algo que deve ser utilizado para somar e incrementar os métodos de ensino e aprendizagem existentes.

\section{Referências}

AKKERMAN, S., ADMIRAAL, W., \& HUIZENGA, J. (2009). Storification in history education: a mobile game in and about medieval Amsterdam. Computers \& Education, 52(2), 449-459. Disponível em: < https://www.sciencedirect.com/science/article/pii/S0360131508001498 >. Acesso em 28/05/2018.

ARAÚJO, R. S. Contribuições da Metodologia WebQuest no Processo de letramento dos alunos nas séries iniciais no Ensino Fundamental. In: MERCADO, L. P. L. (Org.). Vivências com Aprendizagem na Internet. Maceió: Edufal, 2005, p. 11-45. Disponível em: $<$ http://webcache.googleusercontent.com/search?q=cache:WCjw-

mFJKaoJ:www.repositorio.ufal.br/bitstream/riufal/1350/1/Viv\%25C3\%25AAncias\%2520com\%2 520aprendizagem $\% 2520 \mathrm{na} \% 2520 \mathrm{internet} . \mathrm{pdf}+\& \mathrm{~cd}=2 \& \mathrm{hl}=\mathrm{pt}-\mathrm{BR} \& \mathrm{ct}=\mathrm{clnk} \& \mathrm{gl}=\mathrm{de}>$. Acesso em 28/05/2018.

BARROS, G. C. Webquest: metodologia que ultrapassa os limites do ciberespaço. In: Inclusão digital nas escolas públicas: escola software livre. Paraná-Brasil, p. 1-18, nov., 2005. Disponível em: $<w w w . g i l i a n . e s c o l a b r . c o m / t e x t o s / w e b q u e s t \_g i l i a n c r i s . p d f>$. Acesso em: 28/05/2018. 
BILLETT, S. Situated learning: bridging sociocultural and cognitive theorising. Learning and Instruction, 6(3), 263-280, 1996. Disponível em: < https://www.sciencedirect.com/science/article/pii/0959475296000060 >. Acesso em 28/05/2018.

BARBOUR, M. K., THOMAS, G. B., RAUSCHER, D.; RIEBER, L. P. Homemade PowerPoint games. In A. Hirumi (Ed.), Playing games in school: Video games and simulations for primary and secondary education (pp. 333-347). Washington, DC: International Society for Technology in Education. 2010.2 Disponível em: < https://www.academia.edu/2311703/Barbour_M._K._Thomas_G._B._Rauscher_D._and_Rieber_ L._P._2010_._Homemade_PowerPoint_games._In_A._Hirumi_Ed._Playing_games_in_school_V ideo_games_and_simulations_for_primary_and_secondary_education_pp._333-

347_._Washington_DC_International_Society_for_Technology_in_Education>. Acesso em 28/05/2018.

BRASIL. Ministério da Educação. Recursos da Internet para Educação: Webquest. Brasília: MEC, 2015. Disponível em: < http://webeduc.mec.gov.br/webquest/>. Acesso em 28/05/2018.

CHAN, Y.-Y. Teaching queueing theory with an inquiry-based learning approach: a case for applying WebQuest in a course in simulation and statistical analysis. Paper presented at the 37th ASEE/IEEE Frontiers in Education Conference, Milwaukee, WI, October 10-13, 2007. Disponível em: <https://pdfs.semanticscholar.org/5a99/aeac968d16a9d13a071c4ff85084b0cdccd7.pdf>. Acesso em 28/05/2018.

CHANG, CS. The Study on Integrating WebQuest with Mobile Learning for Environmental Education. Computers \& education, Vol. 57, Fasc. 1, p. 1228-1239. Disponível em: < https://wwwsciencedirect-

com.ez24.periodicos.capes.gov.br/science/article/pii/S0360131510003544?via\%3Dihub >. Acesso em 26/05/2018.

CHEN, Y.-S., KAO, T.-C., SHEU, J.-P. A mobile learning system for scaffolding bird watching learning. Journal of Computer Assisted Learning, 19, 347-359. 2003. Disponível em: < http://citeseerx.ist.psu.edu/viewdoc/download?doi=10.1.1.324.151\&rep=rep1\&type=pdf > Acesso em 28/05/2018.

COELHO, L. C. A.; VIDAL, E. M. Análise de Webquests: contribuições da metodologia da problematização. Revista Tecnologias na Educação, Campinas, Ano 1, n./v.1, dez. 2009. Disponível em: < http://tecedu.pro.br/wp-content/uploads/2015/07/Art-2-vol1-dez-2009.pdf>. Acesso em 28/05/2018.

DODGE, B. Some thoughts about WebQuests. The Distance Educator, 1(3), 12-15. 1995. Disponível em: < http://jotamac.typepad.com/jotamacs_weblog/files/WebQuests.pdf >. Acesso em 28/05/2018.

DODGE, B. Some thoughts about WebQuests. Retrieved September. 1997.

DODGE, B. Five rules for writing a great WebQuest. Learning \& Learning with Technology, 28(8), 6-9. 2001. Disponível em: 
https://www.researchgate.net/publication/285664985_Five_rules_for_writing_a_great_WebQuest >. Acesso em 28/05/2018.

DOGRU, M.; SEKER, F.; GENÇOSMAN, T. The effect of use of webquest in science education on success, selfefficacy and web-based education attitudes of primary school students. Practice and Theory in Systems of Education, Vol. 6, N. 4, 2011. Disponível em: < http://epa.oszk.hu/01400/01428/00018/pdf/EPA01428_ptse_1810MustafaFatihTuna.pdf>. Acesso em 28/05/2018.

GASKILL, M., MCNULTY, A., \& Brooks, D. W. (2006). Learning from WebQuests. Journal of Science Education and Technology, 15(2), 133-136. Disponível em: < https://eric.ed.gov/?id=EJ748830>. Acesso em 28/05/2018.

GREENWALD, R., HEDGES, L. V.; LAINE, R. D. The Effect of School Resources on Student Achievement. Research Article. $1996 . \quad$ Disponível em: $<$ http://journals.sagepub.com/doi/abs/10.312/00346543066003361?journalCode=rera $>$. Acesso em 28/05/2018.

KANUKA, H., ROURKE, L., LAFLAMME, E. The influence of instructional methods on the quality of online discussion. British Journal of Educational Technology, 38(2), 260-271. 2007. Disponível em:

http://citeseerx.ist.psu.edu/viewdoc/download?doi=10.1.1.453.4021\&rep=rep1\&type=pdf >. Acesso em 28/05/2018.

KENSKI, V. M. Tecnologias e ensino presencial e a distância. 5. ed. Campinas: Papirus, 2003. (Série Prática Pedagógica)

LAMB, A., \& TECLEHAIMANOT, B. WebQuest theoretical foundations.2004.

PELLICCIONE, D. L., \& Craggs, G. J. WebQuests: an online learning strategy to promote cooperative learning and higher-level thinking. Paper presented at the AARE Conference, 2007. Disponível em: < https://www.aare.edu.au/publications-database.php/5484/webquests-an-onlinelearning-strategy-to-promote-cooperative-learning-and-higher-level-thinking>. Acesso em 28/05/2018.

PIMENTEL, F. S. C. Formação de Professores e Novas Tecnologias: possibilidades e desafios da utilização de webquest e webfólio na formação continuada. 2007. Disponível em: < http://www.companhiadolazer.com.br/pdf/artigo6.pdf>. Acesso em 28/05/2018.

RAMOS, S. Tecnologias da Informação e Comunicação - Conceitos Básicos. Manual da disciplina. Escola Dr. Mário Sacramento, Aveiro, Portugal, 2008.

RAMSEY, J. M., \& HUNGERFORD, H. R. The effects of issue investigation and action training on environmental behaviors in seventh grade students. Journal of Environmental Education, 20(4), $29-34$. 1989.

Disponível em: https://www.tandfonline.com/doi/abs/10.1080/00958964.1989.9943036 >. Acesso em 28/05/2018. 
ROGERS, Y., PRICE, S., RANDELL, C., Fraser, D. S., Weal, M., \& Fitzpatrick, G. Ubi-learning integrates indoor and outdoor experiences. Communications of the ACM, 48(1), 55-59. 2005. Disponível em: < https://dl.acm.org/citation.cfm?id=1039570>. Acesso em 28/05/2018.

SERVIÇO NACIONAL DE APRENDIZAGEM COMERCIAL. WebQuest: O que é. São Paulo, 2003. Disponível em: <http://webquest.sp.senac.br/textos/oque〉. Acesso em 28/05/2018.

SEVERINO, A. J. Metodologia do trabalho científico. 23. ed. São Paulo: Cortez, 2007.

SILVA, F. Q.; FERRARI, H. O. A Webquest como atividade didática potencializadora da educação. Revista Novas Tecnologias na Educação, Porto Alegre, v. 7, n. 1, p. 1-10, jul. 2009. Disponível em: $<$ http://sefarditas.net.br/ava/oficina_online/mest/webquest.pdf >. Acesso em 28/05/2018.

SOUZA, JL; REIS, JIV; DUTRA, A; ROSA VF. A webquest como recurso pedagógico no curso de pedagogia. Texto livre linguagem e tecnologia. Belo Horizonte, v. 10, n. 1, p. 164-177, jan.-jun. 2017.

<http://www.periodicos.letras.ufmg.br/index.php/textolivre/article/view/11031/10485>. Acesso em 28/05/2018.

VIDONI, K. L., \& MADDUX, C. D. WebQuests. Computers in the Schools, 19(1), 101-117. 2002.

WANG, F., \& HANNAFIN, J. M. Integrating Webquests in pre-service teacher education. Educational Media International, 45 (1), 59-73. 2008. Disponível em: < https://www.tandfonline.com/doi/abs/10.1080/09523980701847214? src=recsys\&journalCode=re mi20>. Acesso em 28/05/2018.

WATSON, K. L. (1999). WebQuests in the middle school curriculum: promoting technological literacy in the classroom. Meridian: A Middle School Computer Technologies Journal, 2(2). Disponível em:

http://citeseerx.ist.psu.edu/viewdoc/download?doi=10.1.1.516.4348\&rep=rep1\&type=pdf $\quad>$. Acesso em 28/05/2018.

YODER, M. B. (1999). The student WebQuest: a productive and thought-provoking use of the Internet. Learning \& Leading with Technology, 26(7), 6-9, \& 52.

\section{Como citar este artigo (Formato ABNT):}

GOMES, Gilmária Henllen G.; AMORIM, Liromaria Maria de; RODRIGUES, Micaelle N. D.; BARBOSA, Jailma dos S.; OLIVEIRA, Gislene Farias de. Webquest e mobile-learning como métodos pedagógicos de ensino e aprendizagem: um novo olhar?. Id on Line Revista Multidisciplinar e de Psicologia, 2018, vol.12, n.41, p.154-168. ISSN: 1981-1179.

Recebido: 31.05 .2018

Aceito: 13.06 .2018 